\title{
Travelling solitons in the parametrically driven nonlinear Schrödinger equation
}

\author{
I.V. Barashenkovi, E.V. Zemlyanayal, M. Bärt \\ Max-Planck-Institut für Physik komplexer Systeme, Nöthnitzer Str.38, Dresden, Germany
}

\begin{abstract}
We show that the parametrically driven nonlinear Schrödinger equation has wide classes of travelling soliton solutions, some of which are stable. For small driving strengths stable nonpropagating and moving solitons co-exist while strongly forced solitons can only be stable when moving sufficiently fast.

PACS number(s): 05.45.Yv, 05.45.Xt
\end{abstract}

\section{INTRODUCTION}

The parametrically driven damped nonlinear Schrödinger (NLS) equation,

$$
i \psi_{t}+\psi_{X X}+2|\psi|^{2} \psi-\psi=h \psi^{*}-i \gamma \psi
$$

was used to model the nonlinear Faraday resonance in a vertically oscillating water tank [1,2] and the effect of phase-sensitive parametric amplifiers on solitons in optical fibers [3]. The same equation describes an easy-plane ferromagnet with a combination of a static and hf field in the easy plane [4.5], and the planar weakly anisotropic $X Y$ model [6]. It also serves as a continuum limit for the parametrically driven Frenkel-Kontorova chain (an array of diffusively coupled pendula). The Frenkel-Kontorova system is regarded as a fairly realistic model of a number of physical and biological systems and phenomena, including ladder networks of discrete Josephson junctions, charge-density-wave conductors, crystal dislocations in metals, DNA dynamics and proton conductivity in hydrogen-bonded chains [7].

The undamped undriven nonlinear Schrödinger equation exhibits soliton solutions which can travel with arbitrary velocity and transport physical characteristics such as mass, momentum and energy. (For the sake of brevity, we are making use of the hydrodynamical interpretation of the equation here.) In equation (11), the second term in the right-hand side accounts for dissipative losses which occur in all physical systems. The dissipation has two visible effects on the soliton: it attenuates its speed and damps its amplitude. The main purpose of the introduction of the pumping (represented by the first term in the right-hand side) is to compensate for these losses. The parametric forcing is well known to be capable of counterbalancing the damping of the quiescent soliton's amplitude; a natural question now is whether it can sustain its motion with a nonzero velocity.

In fact the existence of travelling solitons is a nontrivial matter even in the absence of damping. The driving term $h \psi^{*}$ in Eq.(1) breaks the galilean invariance of the unperturbed nonlinear Schrödinger equation and hence one cannot obtain a moving soliton simply by boosting a static one. However the galilean or Lorentz symmetry is not a prerequisite for the existence of moving nonlinear waves. For example, dissipative systems do not possess any symmetries of this kind but are well known to support stably propagating fronts and pulses whose velocities are determined by parameters of the model. In particular, travelling domain walls arise in the parametrically-driven Ginsburg-Landau equations (where the motion is due to nongradient terms) 8. As far as solitons in Hamiltonian systems are concerned, the example of dark solitons in the nonlinear Schrödinger equations suggests that they have even greater mobility than dissipative fronts and pulses. Although in this case the galilean invariance is broken by the presence of the nonvanishing background, the dark solitons can propagate with arbitrary speeds bounded only by the velocity of sound waves 911 .

A number of nonstationary regimes were reported in the water tank experiments, including the formation of oscillating soliton pairs [2], but no steadily moving solitons were detected so far. On the other hand, numerical simulations of the undamped parametrically driven NLS equation [Eq.(1) with $\gamma=0$ ] did exhibit travelling localised objects [12]. It has remained an open question whether these moving objects preserve their speed and amplitude, or attenuate and decay slowly due to the emission of the second-harmonic radiation. The aim of the present paper is to study the existence of steadily propagating solitons, and examine their stability. Here we are confining ourselves to the undamped situation relegating the analysis of the effect of damping to future publications.

In addition to their role in transport phenomena, stably moving solitons are also of interest as alternative attractors which may compete with (static or oscillating) nonpropagating solutions. We will demonstrate that stable travelling solitons do exist in the parametrically driven nonlinear Schrödinger equation. Moreover, there are parameter ranges where moving solitons are stable whereas their quiescent counterparts are not. Unstable solitons are not meaningless either; they arise as long-lived transients and intermediate states in spatiotemporal chaotic regimes. In this paper we will identify oscillatory and translational instabilities of travelling solitons and simulate their nonlinear evolution near the transition curves.

The structure of the paper is as follows. In section II 
we derive an apriori bound for the existence domain of travelling solitons and introduce the linearised eigenvalue problem for their stability analysis. We also discuss some general properties of eigenvalues and eigenfunctions and formulate a simple criterion for the onset of the nonoscillatory instability: $\partial P / \partial V=0$, where $V$ is the velocity of the steadily moving soliton, and $P$ the associated momentum.

In section III we present several explicit quiescent $(V=0)$ solutions, including a stationary complex of two solitons, and then derive the necessary condition for a static solution to be continuable to nonzero velocities. This condition is that the motionless solution should either not have any "free" parameters apart from the translational shift, or, if there is an additional parameter $z$, the equation $\partial P / \partial z=0$ should be satisfied. Here $P$ is the momentum of the motionless localised solution (which, contrary to one's mechanical intuition, is not necessarily equal to zero). There are three static solutions satisfying the above condition, two of which being the well-known constant-phase $\psi_{+}$and $\psi_{-}$solitons, respectively, while the third solution looks like a pulse with a bell-shaped modulus and twisted phase.

The most important results of this work are contained in section IV] where we report on the numerical continuation of various branches of solutions and their stability analysis. In agreement with the analytical predictions of the preceding section, we find that each of the above static solutions admits the continuation to nonzero $V$. The stability properties of travelling solitons result from an intricate interplay of two types of instability, the oscillatory and translational instability. In accordance with the conclusions of section II, the numerical analysis of the linearised eigenvalues shows that the transition curves of the translational instability satisfy $\partial P / \partial V=0$. One interesting conclusion of the stability analysis is that although quiescent solitons are unstable for driving strengths larger than $h=0.064$, there are stable moving solitons for any $0 \leq h \leq 1$. We discuss in detail the soliton's transformation as it is continued in $V$, paying special attention to the dynamics of the associated linearised eigenvalues on the complex plane. Two different scenarios of the transformation are identified, one occurring for small $h$ and the other one for larger driving strength, and we also describe an interesting cross-over from one to another.

Section $\mathrm{V}$ is devoted to the direct numerical simulations of the full time-dependent nonlinear Schrödinger equation. We show that the evolution of both types of the soliton instability leads, as $t \rightarrow \infty$, to the same asymptotic attractors. Finally, section VI contains concluding remarks and outlines some open problems.

\section{STEADILY TRAVELLING WAVES: EXISTENCE AND STABILITY}

\section{A. Existence domain and integrals of motion}

We will confine ourselves to localised travelling waves of the simplest form, $\psi(X, t)=\psi(X-V t)$. Transforming to the moving frame, these correspond to timeindependent soliton solutions of the equation

$$
i \psi_{t}-i V \psi_{x}+\psi_{x x}+2|\psi|^{2} \psi-\psi=h \psi^{*},
$$

where $x=X-V t$. We will search for these static solutions by solving an ordinary differential equation

$$
-i V \psi_{x}+\psi_{x x}+2|\psi|^{2} \psi-\psi=h \psi^{*}
$$

under the vanishing boundary conditions $|\psi(x)| \rightarrow 0$ as $|x| \rightarrow \infty$. Here $h$ is always taken positive; negative $h$ 's can be recovered by the phase transformation $\psi \rightarrow i \psi$.

If $\psi(x)$ is a solution, then so is $-\psi(x)$. Next, it is straightforward to notice that if the function $\psi(x)$ describes a soliton travelling with the velocity $V$, the function $\psi^{*}(\mathrm{x})$ describes a soliton moving with the velocity $-V$. We will try to restrict ourselves to positive $V$ 's wherever possible; we will only present negative velocities where this may help visualising how different branches of solutions are connected. Since the soliton moving with the velocity $-V$ is given by $\psi(-x)$, the above observation tells us that either $\psi^{*}(x)= \pm \psi(-x)$ (that is, one of the real and imaginary part of the solution is even and the other one odd), or there are two solutions associated with the same $V$. (Here we are not making any difference between solutions which are different just in the overal sign.) In the latter case the solutions will not exhibit the $\psi^{*}(x)= \pm \psi(-x)$ symmetry.

Next, it is easy to show that solitons cannot travel faster than a certain limit speed. Indeed, as $|x| \rightarrow \infty$, the soliton's asymptotic tail decays as $\psi(x) \sim e^{-\kappa x}$, where

$$
2 \kappa^{2}=2-V^{2} \pm \sqrt{\left(2-V^{2}\right)^{2}+4\left(h^{2}-1\right)} .
$$

Large driving strengths, $h>1$, are of little interest to us as in this case the zero background, $\psi(x)=0$, is unstable with respect to continuous spectrum waves [5]. Therefore we are not going to discuss this case here. In the complementary region $h<1$, the complex structure of $\kappa$ depends on the value of the velocity. When $V^{2}<2-2 \sqrt{1-h^{2}}$, there are four real exponents; for $2-2 \sqrt{1-h^{2}}<V^{2}<c^{2}$, where

$$
c=\sqrt{2+2 \sqrt{1-h^{2}}}
$$

we have a quadruplet of complex $\kappa$ 's, and finally, for $V^{2}>c^{2}$ all four exponents are imaginary. Consequently, there can be no exponentially localised solitons travelling faster than $c$. Physically, $c$ represents the minimum phase velocity of linear waves governed by Eq.(11), and our condition $V<c$ is essentially an exclusion principle ruling out a resonance between solitons and linear waves.

In the undamped case the parametrically driven NLS equation (2) conserves the momentum, 


$$
P=\frac{i}{2} \int\left(\psi_{x}^{*} \psi-\psi_{x} \psi^{*}\right) d x
$$

and energy:

$$
E=\operatorname{Re} \int\left(\left|\psi_{x}\right|^{2}+|\psi|^{2}-|\psi|^{4}+h \psi^{2}\right) d x .
$$

Noting that stationary solutions satisfy

$$
\left|\psi_{x}\right|^{2}=|\psi|^{2}-|\psi|^{4}+h \operatorname{Re} \psi^{2},
$$

Eq.(目) can be rewritten as

$$
E=2 \operatorname{Re} \int\left(|\psi|^{2}-|\psi|^{4}+h \psi^{2}\right) d x .
$$

Containing no derivatives, this formula for energy of stationary solutions has obvious advantages for the numerical implementation.

\section{B. Linearised eigenvalue problem}

In this paper we solve the equation (3) numerically and examine the stability of the resulting solutions by studying the associated eigenvalue problem. This eigenvalue problem arises by assuming a small perturbation of the form

$$
\delta \psi(x, t)=y(x) e^{\lambda t}, \quad y(x)=\delta u(x)+i \delta v(x) .
$$

Substituting into (2) gives

$$
\mathcal{H} Y=\lambda J Y,
$$

where the hermitean operator $\mathcal{H}$ has the form

$$
\begin{array}{r}
\mathcal{H}=I\left(-\partial_{x}^{2}+1\right)+V J \partial_{x}+ \\
+\left(\begin{array}{lr}
h-6 u^{2}-2 v^{2} & -4 u v \\
-4 u v & -h-6 v^{2}-2 u^{2}
\end{array}\right) ;
\end{array}
$$

the matrix $J$ is given by

$$
J=\left(\begin{array}{rr}
0 & -1 \\
1 & 0
\end{array}\right),
$$

and the column-vector $Y(x)=(\operatorname{Re} y, \operatorname{Im} y)^{T}=(\delta u, \delta v)^{T}$. In Eq.(10) $I$ is the identity matrix, and we have decomposed the stationary solution as $\psi(x)=u(x)+i v(x)$.

For symmetric solutions satisfying $\psi^{*}(x)= \pm \psi(-x)$ eigenvalues will always come in $(\lambda,-\lambda)$-pairs. This follows from the fact that for these solutions changing $x \rightarrow-x$ in the operator (10) amounts to changing the sign of its off-diagonal elements, and hence if $(\delta u(x), \delta v(x))^{T}$ is an eigenfunction associated with an eigenvalue $\lambda$, the column $(\delta u(-x),-\delta v(-x))^{T}$ will serve as an eigenfunction associated with an eigenvalue $-\lambda$. As far as the zero eigenvalue is concerned, it will have a twin with the eigenfunction $(\delta u(-x),-\delta v(-x))^{T}$ unless its eigenfunction $y=\delta u+i \delta v$ satisfies the symmetry $y^{*}(x)=e^{i \varphi} y(-x)$, where $\varphi=$ const.

To complete the discussion of the spectrum structure, we mention that there are two branches of the continuous spectrum lying on the imaginary axis of $\lambda: \lambda=i \omega_{1,2}(k)$, where

$$
\omega_{1,2}(k)=V k \pm \sqrt{\left(k^{2}+1\right)^{2}-h^{2}},
$$

and $-\infty<k<\infty$. (We are still assuming $h<1$ ). In the region $V^{2}<c^{2}$ which is of interest to us, the continuous spectrum has a gap: $\omega_{1}(k)>\omega_{0}, \omega_{2}(k)<-\omega_{0}$, where $\omega_{0}>0$. This gap can harbour discrete eigenvalues representing stable oscillation modes.

\section{Translational (nonoscillatory) instabilities}

The aim of this subsection is to demonstrate that a pair of pure imaginary eigenvalues can collide at $\lambda=0$ and move onto the real axis only at the point where $\partial P / \partial V=0$. This criterion is known in the context of dark solitons of the undriven nonlinear Schrödinger equations; see [10], [11]. Here we simply adapt the proof given in 10 to the case of the equation with the parametric forcing. An important assumption that we make here, is that the solution whose stability is being examined, does not have any free parameters apart from the trivial translation parameter, $x_{0}$.

First of all we need to make a remark on the integrable case, $h=0$. In this case solutions of the ODE (3) can be obtained from a quiescent soliton of Eq.(11) by a Galilei transformation:

$$
\psi(x)=e^{i(V / 2) x} A \operatorname{sech} A x,
$$

where $A=\sqrt{1-V^{2} / 4}$. For $h=0$ and any $|V|<2$, the linearised operator $\mathcal{H}$ has four zero eigenvalues associated with two eigenvectors. One of these eigenvectors originates from the translation symmetry and the other one results from the phase invariance of Eq.(33). The term $h \psi^{*}$ breaks the phase invariance and hence as $h$ is increased from zero, one pair of eigenvalues $(\lambda,-\lambda)$ moves away from the origin on the complex plane. As $h$ and $V$ are further varied, a pair of eigenvalues may return to the origin. If the solution of Eq.(3) at the point of their return is a member of a family parametrised by two free parameters, we will have, again, four zero eigenvalues with two eigenfunctions. (The eigenfunctions are simply derivatives of the solution with respect to the free parameters.) Our analysis will not be applicable in this case, and the equality $\partial P / \partial V=0$ does not have to be valid at the return point. (We will come across this type of a situation in section IV D below.) However, a more common situation is when the solution at the return point is a member of a one-parameter family. We will show that in this case the relation $\partial P / \partial V=0$ does have to be in place. 
Let us denote $V_{c}$ the velocity for which the eigenvalue of the operator (9)-(10) vanishes. We can develop the solution $\psi(V ; x)$ in powers of $\epsilon=V-V_{c}$ :

$$
\psi(V ; x)=\psi_{0}(x)+\epsilon \psi_{1}(x)+\epsilon^{2} \psi_{2}(x)+\ldots,
$$

where $\psi_{0}=\psi\left(V_{c} ; x\right)$. Accordingly, the operator $\mathcal{H}$ expands as $\mathcal{H}=\mathcal{H}_{0}+\epsilon \mathcal{H}_{1}+\epsilon^{2} \mathcal{H}_{2}+\ldots$ If the eigenvalue $\lambda$ moves from imaginary to the real axis, it is natural to assume that it admits an expansion of the form

$$
\lambda=\epsilon^{1 / 2} \lambda_{1}+\epsilon^{3 / 2} \lambda_{3}+\epsilon^{5 / 2} \lambda_{5}+\ldots
$$

The associated eigenfunction is then developed as

$$
Y(x)=Y_{0}(x)+\epsilon^{1 / 2} Y_{1}(x)+\epsilon Y_{2}(x)+\ldots
$$

When $\epsilon=0$, we have $\mathcal{H}_{0} Y_{0}=0$, i.e. $Y_{0}$ is a null eigenvector at the bifurcation point $V=V_{c}$. Since we have assumed that $\psi\left(V_{c}, x\right)$ is a member of a one-parameter family of solutions, the operator $\mathcal{H}_{0}$ has only one null eigenvector, and we have to identify $Y_{0}=\Psi_{0}^{\prime}(x)$. Here $\Psi_{0}$ is a column-vector formed by the real and imaginary part of the soliton $\psi_{0}: \Psi_{0}=\left(u_{0}, v_{0}\right)^{T}$. The prime indicates differentiation with respect to $x$.

Next, setting the coefficient of $\epsilon^{1 / 2}$ to zero yields

$$
\mathcal{H}_{0} Y_{1}=\lambda_{1} J Y_{0}
$$

Comparing this to the equation

$$
\left.\mathcal{H}_{0} \frac{\partial \Psi}{\partial V}\right|_{V=V_{c}}=-J \Psi_{0}^{\prime},
$$

which arises from the differentiation of Eq.(3) with respect to $V$, we get

$$
Y_{1}(x)=-\left.\lambda_{1} \frac{\partial \Psi}{\partial V}\right|_{V=V_{c}} .
$$

(In the above equations $\Psi=(u, v)^{T}$.) The coefficient of $\epsilon^{1}$ produces

$$
\mathcal{H}_{0} Y_{2}=\lambda_{1} J Y_{1}-\mathcal{H}_{1} Y_{0}
$$

which has bounded solutions if the right-hand side is orthogonal to the null eigenvector of $\mathcal{H}_{0}$ :

$$
\lambda_{1} \int Y_{0} J Y_{1} d x-\int Y_{0} \mathcal{H}_{1} Y_{0} d x=0 .
$$

The second term in equation $(15)$ is readily shown to vanish - one only needs to expand the identity $\mathcal{H} \Psi^{\prime}=0$ in $\epsilon$. (The coefficient of $\epsilon^{1}$ gives $\mathcal{H}_{1} \Psi_{0}^{\prime}=-\mathcal{H}_{0} \Psi_{1}^{\prime}$. Taking the scalar product with $\Psi_{0}^{\prime}$ yields the required $\int \Psi_{0}^{\prime} \mathcal{H}_{1} \Psi_{0}^{\prime} d x=0$.) On the other hand, the first term in Eq.(15) is equal to $\left(\lambda_{1}^{2} / 2\right) \partial P / \partial V$. Consequently, Eq.115) gives either $\partial P / \partial V=0$ or $\lambda_{1}=0$. If we assume that $\lambda_{1}=0$, we will not be able to conclude that $\partial P / \partial V=0$ at this order of the expansion. However, the order $\epsilon^{2}$ will then give us $\lambda_{2}^{2} \partial P / \partial V=0$, which implies either $\partial P / \partial V=0$ or $\lambda_{2}=0$. Proceeding by a similar token we will eventually arrive at the equation $\partial P / \partial V=0$ at some order $\epsilon^{n}$ where $n$ is such that $\lambda_{n} \neq 0$. (Alternatively, we will have to conclude that all $\lambda_{n}=0$ and hence we are dealing with a symmetry eigenvalue which is equal to zero for all $V$.)

Thus a pair of real or pure imaginary eigenvalues of the same magnitude and opposite sign, can only collide for the value of $V$ which satisfies $\partial P / \partial V=0$. Here we wish to re-emphasise that we have obtained this conclusion under the assumption that the geometric multiplicity of the zero eigenvalue is not increased at the point of collision. A simple example when this assumption is not valid, is furnished by the case $h=0$. In this case the momentum corresponding to the soliton (12) is given by $P=V \sqrt{1-V^{2} / 4}$. Although $P$ has a maximum for $V=\sqrt{2}$, the stability properties of the undriven soliton do not change at this point. The reason is that for each $V$ the operator $\mathcal{H}$ has two null eigenvectors in this case, and hence we cannot make the identification $Y_{0}=\Psi_{0}^{\prime}$. (Instead, $Y_{0}$ will be a linear combination of two zero modes.) Consequently, the above proof becomes invalid in this case.

Finally, one can easily check that the above result does not really depend on how the eigenvalue $\lambda$ expands in powers of $\epsilon$. We assumed that the expansion (13) starts with terms of order $\epsilon^{1 / 2}$. This assumption is natural and supported by the numerical evidence; however, even if we had postulated the expansion starting with terms of order $\epsilon^{1 / 4}, \epsilon^{1 / 3}$ or say, $\epsilon$, we would have still arrived at the same necessary condition for the zero crossing: $\partial P / \partial V=0$.

\section{QUIESCENT SOLUTIONS $(V=0)$}

\section{A. The "twist" soliton}

In order to continue in $V$ it is useful to have some starting solutions for $V=0$. Two such stationary solutions are given by

$$
\begin{gathered}
\psi_{+}(x)=A_{+} \operatorname{sech}\left(A_{+} x\right), \\
\psi_{-}(x)=i A_{-} \operatorname{sech}\left(A_{-} x\right),
\end{gathered}
$$

where $A_{ \pm}^{2}=1 \pm h$. The soliton $\psi_{-}$is unstable with respect to a nonoscillatory mode for all $h$ [5]. The $\psi_{+}$is stable for $h<h_{0}=0.063596$ but developes an oscillatory instability as $h$ is increased beyond $h_{0}$ [5],12].

In this section we will produce several more explicit solutions of the undamped, parametrically driven NLS equation (2). Writing $\psi=u+i v$, the stationary equation (3) transforms into the system

$$
\begin{gathered}
u_{x x}-u-h u+2 u\left(u^{2}+v^{2}\right)=0, \\
v_{x x}-v+h v+2 v\left(u^{2}+v^{2}\right)=0 .
\end{gathered}
$$


We can try to find explicit solutions of this system by imposing some plausible reductions, for instance by identifying $u^{2}+v^{2}$ with a function of $u$ and its derivatives: $u_{x}, u_{x x}$ and so on. In this case Eq.(17) is an equation for $u$ only, while Eq.(18) becomes a linear equation with variable coefficients. The simplest choice $u^{2}+v^{2}=C u^{2}$ leads to the $\psi_{+}$and $\psi_{-}$solitons (16). Another simple possibility is to require that

$$
u^{2}+v^{2}=C u, \quad C=\text { const; }
$$

this converts the first equation into the stationary $\mathrm{KdV}$ with the well-known localised solution

$$
u=\frac{3}{4} \frac{1+h}{C} \operatorname{sech}^{2} \xi, \quad \xi=\frac{\sqrt{1+h}}{2} x .
$$

The second equation has now the form of an eigenvalue problem for the Pöschl-Teller potential:

$$
\left(-\partial_{\xi}^{2}+1-6 \operatorname{sech}^{2} \xi\right) v=E v
$$

where $E=1+4(h-1) /(h+1)$. The operator (20) has two localized eigenfunctions, $v_{0}=\alpha \operatorname{sech}^{2} \xi$ associated with an eigenvalue $E_{0}=-3$, and $v_{1}=\alpha \operatorname{sech} \xi \tanh \xi$ with an eigenvalue $E_{1}=0$. The first eigenfunction can satisfy the constraint (19) for no $\alpha$ while the second one will satisfy it if we set $C^{2}=\alpha^{2}=\frac{3}{4}(1+h)$.

Noticing that $E=0$ corresponds to $h=\frac{3}{5}$, we conclude that for $h=\frac{3}{5}$ there is an explicit solution of the form

$$
u_{T}=\sqrt{\frac{6}{5}} \operatorname{sech}^{2} \xi ; \quad v_{T}= \pm \sqrt{\frac{6}{5}} \operatorname{sech} \xi \tanh \xi,
$$

where $\xi=\sqrt{\frac{2}{5}} x$. Similarly to the solitons $\psi_{+}$and $\psi_{-}$, the modulus of the above solution is bell-shaped, but, unlike the constant phase of the $\psi_{+}$and $\psi_{-}$solitons, the phase of this solution varies. (In the case of the positive sign in (21) the phase grows from $-\pi / 2$ at $x=-\infty$ to $\pi / 2$ at $x=\infty$.) The solution looks like a pulse twisted by $180^{\circ}$ in the $(u, v)$-plane. For this reason we will be referring to Eq.(21) as the "twist" soliton.

\section{B. The twist soliton as a bound state}

The system (17)-(18) appeared previously as a stationary system governing light pulses in a birefringent optical fiber. Using Hirota's approach, Tratnik and Sipe 13 have obtained the following exact solution to eqs.(3),(17)(18):

$$
\begin{array}{r}
\psi=\psi(z ; x)=u+i v, \\
u=2 A_{+} e^{\theta_{2}} D^{-1}\left(1+e^{2\left(\theta_{1}-\beta\right)}\right), \\
v=2 A_{-} e^{\theta_{1}} D^{-1}\left(1-e^{2\left(\theta_{2}-\beta\right)}\right),
\end{array}
$$

where
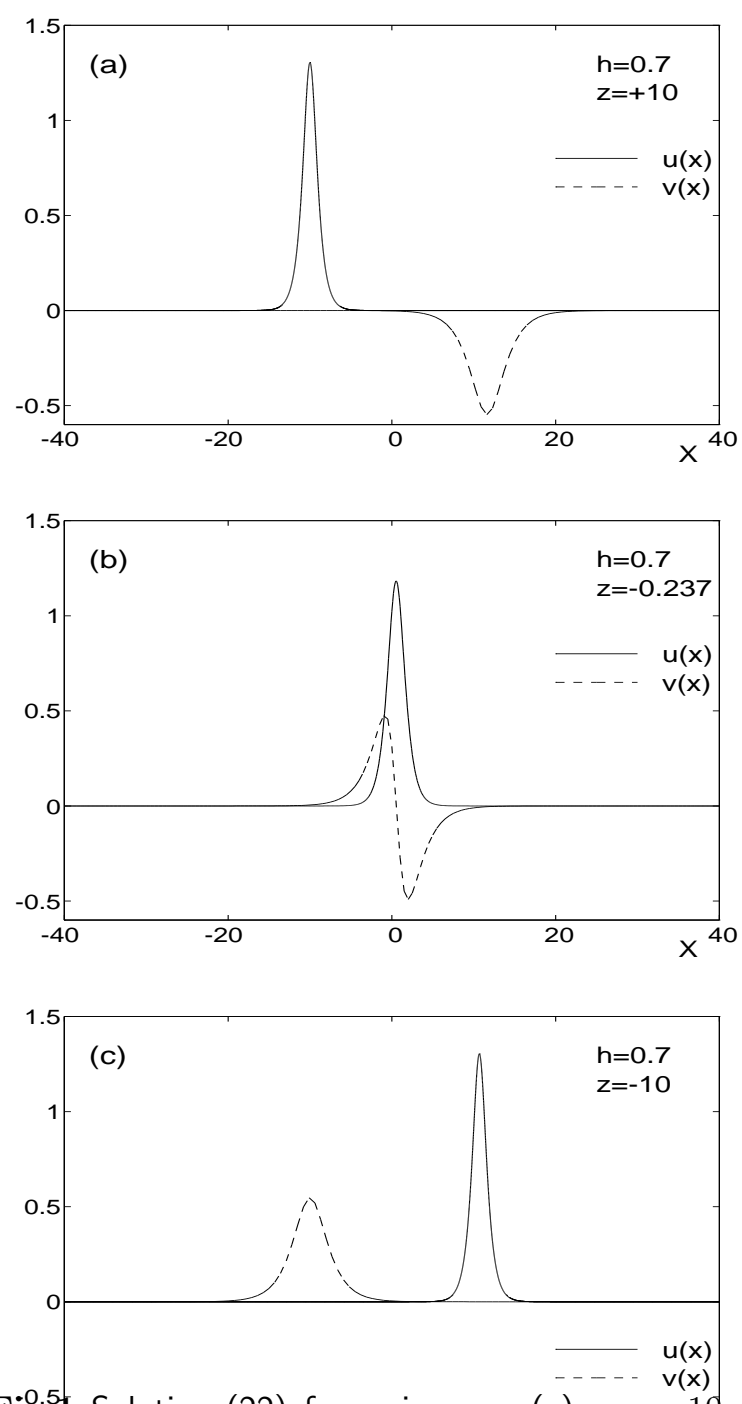

Fig:-1. Solution (22) for various $z$. (a): $z=10$. (b): $z=\zeta$ with $\zeta$ as in (25) (the twist soliton); (c): $z^{\mathrm{X}=}=-10$. Solid curve: real part; dashed line: imaginary part.

$$
\begin{aligned}
& D=1+e^{2 \theta_{1}}+e^{2 \theta_{2}}+e^{2\left(\theta_{1}+\theta_{2}-2 \beta\right)}, \\
& \theta_{1}=A_{-}(x-z), \quad \theta_{2}=A_{+}(x+z)
\end{aligned}
$$

the constant

$$
\beta=\frac{1}{2} \ln \left(\frac{A_{+}+A_{-}}{A_{+}-A_{-}}\right)>0,
$$

the amplitudes $A_{ \pm}$are as in (16): $A_{ \pm}=\sqrt{1 \pm h}$, and $z$ is a real parameter which can take arbitrary values. The solution (22) with $z=10$ and -10 is plotted in Fig.1, (a) and (c). As is clear from the figure, for sufficiently large $|z|$ the solution represents a complex of two solitons, $\psi_{+}$ and $\psi_{-}$, with the separation approximately equal to $2|z|$. It is perhaps worth emphasizing here that the parameter $z$ has nothing to do with shifting the solution as a whole, 
$x \rightarrow x-x_{0}$, which is possible due to the translation invariance of Eqs.(11)-(3). (This overall shift parameter, $x_{0}$, is disregarded in equations (21)-(22) and in the remainder of this text). The parameter $z$ is nontrivial in the sense that the shape of the solution depends on $z$.

It is straightforward to verify that our "twist" solution is a particular case of Eq. (22) with $h=\frac{3}{5}$. Indeed, choosing $z=-\frac{1}{8} \sqrt{\frac{5}{2}} \ln 3$ in Eqs.(22), we obtain the soliton (21) (the one with the negative sign), centered at $x=-3 z$. Since the twist is a symmetric solution with sech-shaped modulus, it would be difficult to interpret it as a bound state of the $\psi_{+}$and $\psi_{-}$without embedding it into a broader family of solitonic complexes. In fact, a similar symmetric solution exists for any $h$, not only for $h=\frac{3}{5}$. To see this, we notice a simple relation between two solutions of the form (22) - one with the parameter value $z=\zeta+\xi$ and the other one with $z=\zeta-\xi$ :

$$
\psi(\zeta+\xi ; \eta-y)=\psi^{*}(\zeta-\xi ; \eta+y)
$$

Here $\zeta$ and $\eta$ are defined by the driving strength $h$ :

$$
\zeta=-\frac{\beta}{2}\left(\frac{1}{A_{-}}-\frac{1}{A_{+}}\right)<0
$$

and

$$
\eta=\frac{\beta}{2}\left(\frac{1}{A_{-}}+\frac{1}{A_{+}}\right),
$$

while $\xi$ and $y$ can take arbitrary values. The relation 24 implies that the solution (22) with $z=\zeta$ is symmetric about the point $x=\eta$ :

$$
\psi(\zeta ; \eta-y)=\psi^{*}(\zeta ; \eta+y)
$$

That is, the real part of this solution is even and imaginary part odd with respect to $x=\eta$ :

$$
u(\eta-y)=u(\eta+y), \quad v(\eta-y)=-v(\eta+y) .
$$

(See Fig.1 (b).) This particular representative of the family (22) will play a special role in what follows. For the ease of reference we are retaining the name "twist" for this symmetric solution - for all $h$.

\section{The moving soliton bifurcation}

Suppose the equation (3) has a one-parameter family of quiescent solutions $\psi(z ; x)$. Here $z$ can be any nontrivial parameter; the only requirement is that $z$ should not be just an overall shift in $x$. One such family is given by Eq.22) and there can also be other families for which $\psi$ is not available explicitly. We will show in this section that in order for a solution with some $z=z_{0}$ to be continuable to nonzero $V$, the corresponding momentum integral should satisfy

$$
\left.\frac{\partial P}{\partial z}\right|_{z=z_{0}}=0
$$

Let us assume that Eq.(3) with $V \neq 0$ has a solution $\psi(x)$, and that this solution is an analytic function of $V$ in some neighbourhood of $V=0$. Then we can expand it in the Taylor series

$$
\psi(x)=\psi_{0}(x)+V \psi_{1}(x)+V^{2} \psi_{2}(x)+\ldots,
$$

where $\psi_{0}(x)=\psi\left(z_{0} ; x\right)=u_{0}+i v_{0}$ is some representative of the family of "motionless" solutions $\psi(z ; x)$ with the parameter value $z_{0}$. Substituting (29) into (3) and equating coefficients of like powers of $V$, we get, at the order $V^{1}$ :

$$
\mathcal{H}\left(\begin{array}{l}
u_{1} \\
v_{1}
\end{array}\right)=J \partial_{x}\left(\begin{array}{l}
u_{0} \\
v_{0}
\end{array}\right) .
$$

Here $u_{1}+i v_{1}=\psi_{1}$ and the operator $\mathcal{H}$ is given by Eq.(10). The equation (30) is solvable in the class of square integrable functions if the vector in the right-hand side is orthogonal to all homogeneous solutions, i.e. to all null eigenvectors of the operator $\mathcal{H}$. Since there is a family of "motionless" solutions parametrized by $z$ and by an arbitrary spatial shift $x_{0}$ (which we have disregarded so far), the operator $\mathcal{H}$ has two zero modes. One is the translation mode $\partial_{x} \psi_{0}=\partial_{x}\left(u_{0}+i v_{0}\right)$; the corresponding solvability condition is trivially satisfied:

$$
\int \partial_{x}\left(u_{0}, v_{0}\right) J \partial_{x}\left(\begin{array}{l}
u_{0} \\
v_{0}
\end{array}\right) d x=0 .
$$

The other zero mode is given by the derivative $\partial_{z} \psi_{0}=$ $\partial_{z} u_{0}+i \partial_{z} v_{0}$. The associated solvability condition reads

$$
0=\int\left(\partial_{z} u_{0}, \partial_{z} v_{0}\right) J \partial_{x}\left(\begin{array}{c}
u_{0} \\
v_{0}
\end{array}\right) d x=-\frac{1}{2} \frac{\partial P}{\partial z},
$$

where $P$ is the momentum integral (6). Consequently, a solution with nonzero $V$ can only detach from the $V=0$ branch at the point where $\partial P / \partial z=0$.

Coming back to our explicit solutions, the $\psi_{+}$and $\psi_{-}$ solitons do not have any free parameters apart from the trivial position shift. Consequently, both solutions are continuable to nonzero $V$. Next, we have a family of solitonic complexes (22) with a nontrivial parameter $z$. As one can easily check, the momentum of the complex (22) as a function of $z$ has a single minimum for some finite $z=z_{0}$ and tends to zero as $z \rightarrow \pm \infty$. To find $z_{0}$, we notice that the relation (24) implies

$$
P(\zeta+\xi)=P(\zeta-\xi) \text {. }
$$

This means that the function $P(z)$ is even with respect to the point $z=\zeta$ and therefore, $\zeta$ is the point of the minimum: $z_{0}=\zeta$. Thus, the only representative of the family of the two-soliton complexes 22 that can be continued to nonzero $V$, is our twist soliton, $\psi(\zeta ; x)$. (To be more precise, there are two twist solutions, one with positive and the other one with negative momentum. This is related to the fact that when $V=0$, we can generate new solutions to the system (17)-(18) by changing the sign of just one component $u$ or $v$.) 


\section{BIFURCATION DIAGRAM}

We used a predictor-corrector continuation algorithm with a fourth-order Newtonian solver to continue solutions of equation (3) in $V$. Since derivatives of the momentum integral (6) determine stability and branching properties of solutions, the momentum was our natural choice for the bifurcation measure. Eq.(3) was solved under the vanishing boundary conditions $\psi( \pm L / 2)=0$. We used $L=200$ (except the cases where we had to extend the interval to account for slow decay of solutions) and the discretisation stepsize $\Delta x=0.005$. The eigenvalue problem (9) was solved on the interval $(-50,50)$. Here we utilised the Fourier method, typically with 600 harmonics.

\section{A. The travelling $\psi_{-}$soliton}

We start our description with the branch departing from the quiescent soliton $\psi_{-}$. For every $h$ this branch continues all way to $V=c$, where $c$ is the minimum phase velocity of linear waves given by Eq.(5). (See Fig.2). As $V \rightarrow c$, the decay rate of $\psi(x)$ decreases and the soliton merges with the zero solution, with the momentum $P$ tending to zero. We plotted $P(V)$ for $h=0.1,0.3,0.5,0.7$ and 0.9 in Fig.2. For technical reasons we could not connect the curve $P(V)$ to zero although we were able to approach the value $V=c$ as close as the fourth digit after the decimal point. (The problem is that since the decay rate of the solution decreases, one has to increase the length of the integration interval - and this cannot be done indefinitely.) The only curve which is connected to zero in Fig.2, is the one for the undriven case $h=0$. In this case we enjoy an explicit solution (12) with the momentum $P=V \sqrt{1-V^{2} / 4}$.

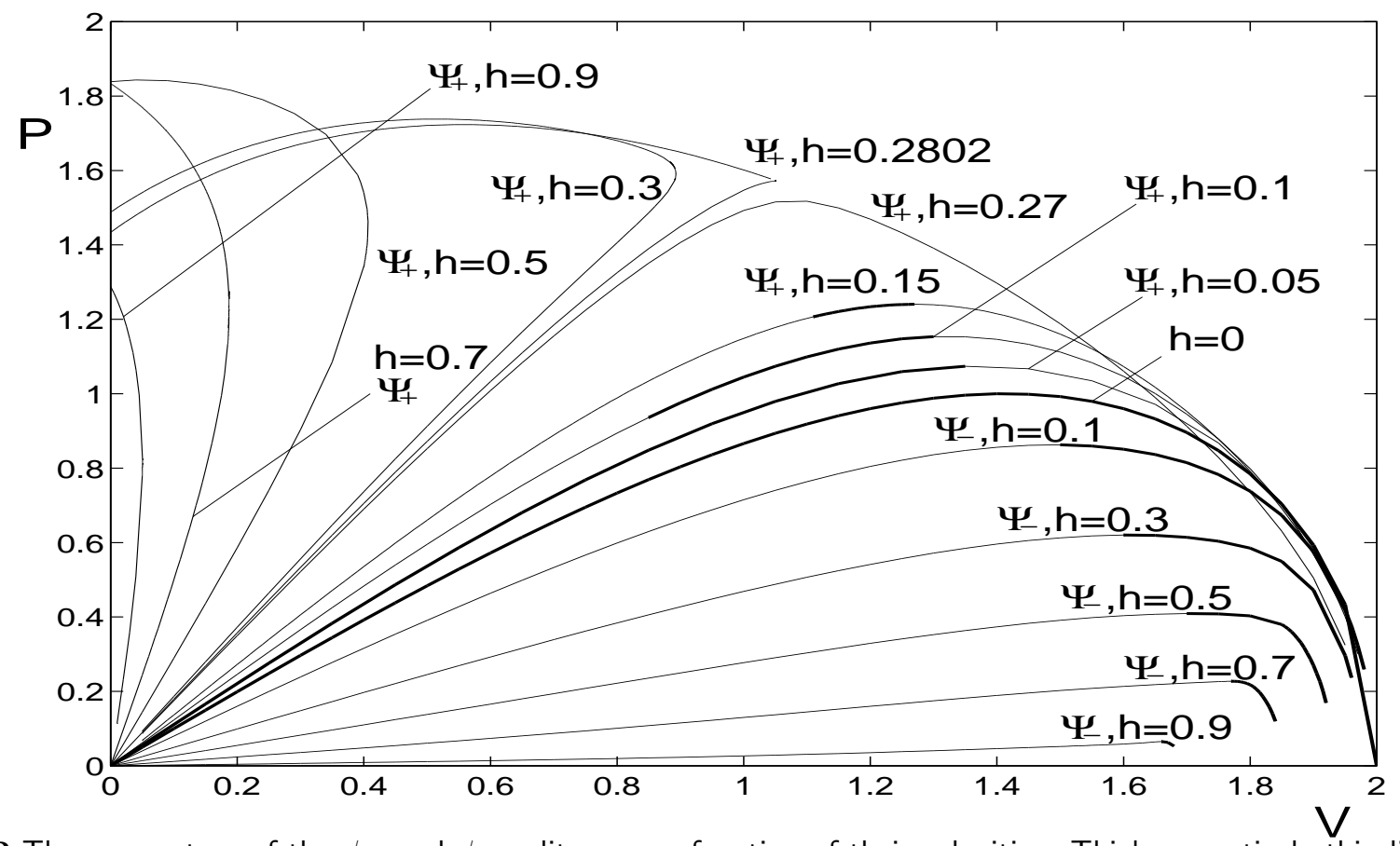

Fig.2 The momentum of the $\psi_{+}$and $\psi_{-}$solitons as a function of their velocities. Thick respectively thin lines depict stable respectively unstable solutions. Note that as $h \rightarrow 0$, the stability domain of the $\psi_{\text {- }}$ tends to $\sqrt{2} \leq V \leq 2$ and that of the $\psi_{+}$to $0 \leq V \leq \sqrt{2}$. The whole of the $h=0$ branch is stable.

For each $h$ the momentum of the soliton has a single maximum on this branch, at $V=V_{c}$ (Fig.2). To the left of $V_{c}$ the linearised operator (9)-(10) has a pair of real eigenvalues $\pm \lambda$ and consequently, the soliton $\psi_{-}$, which is well known to be unstable for $V=0$ [5], remains unstable for small nonzero velocities. As $V$ approaches $V_{c}$, the two eigenvalues converge at the origin on the complex plane, with the associated eigenfunctions tending to the translation mode $\Psi_{0}^{\prime}(x)$. Increasing $V$ past $V_{c}$, the eigenvalues move onto the imaginary axis and hence the $\psi_{\text {- soliton becomes stable for sufficiently large velocities }}$ (where $\partial P / \partial V<0$ ). This change of stability proper- 
ties is in exact agreement with the scenario described in section II C.

\section{B. The travelling $\psi_{+}$soliton; $h \leq 0.25$}

In agreement with conclusions of section III C, we have found that the soliton $\psi_{+}$is also continuable to nonzero velocities. Unlike the $\psi_{-}$-branch where the final product of the continuation process does not depend on the value of $h$, the transformation scenario of the soliton $\psi_{+}$is different for different $h$. For $h<0.28$ the fate of the soliton $\psi_{+}$is similar to that of the $\psi_{-}$. As $V \rightarrow c$, the soliton develops oscillations on its tails; the width of the resulting oscillatory "wavepacket" grows and the amplitude decreases, until the solution becomes equal to zero everywhere. The momentum $P(V)$ tends to zero as $V \rightarrow c$ and has a single maximum at some $V=V_{c}$. Stability properties of the $\psi_{+}$soliton depend on whether $h$ is smaller than 0.064 , lies between 0.064 and 0.25 , or is greater than 0.25 .

Let, first, $h \leq 0.25$. As $V$ is increased to $V_{c}$, a pair of imaginary eigenvalues $\pm \lambda$ collides at the origin on the complex plane and moves onto the real axis. This is in contrast to the $\psi_{-}$soliton, where two real eigenvalues converged at the origin as $V$ was increased. For $V>V_{c}$ (i.e. where the slope $\partial P / \partial V$ is negative), the $\psi_{+}$soliton becomes unstable. (Note that the $\psi_{-}$was unstable for positive slopes $\partial P / \partial V$.) As in the $\psi_{-}$case, this is the translational, or nonoscillatory, instability. Since, as we checked, the eigenfunctions associated with the colliding imaginary eigenvalues tend to $\Psi_{0}^{\prime}(x)$ as $V \rightarrow V_{c}$, this instability is of the type analysed in section II G. The numerically detected instability boundary, defined by the condition $\partial P / \partial V=0$, is in exact correspondence with our analytical predictions.

In addition, for some $V$ the $\psi_{+}$soliton exhibits the oscillatory instability. (The oscillatory instability sets in when four eigenvalues collide, pairwise, on the imaginary axis and emerge into the complex plane.) For $h \leq 0.064$ the oscillatory instability does not arise for any $V$ and hence the whole range $0<V<V_{c}$ is stable. (See the $h=0.05$ curve in Fig.2). For $0.064<h \leq 0.25$ the oscillatory instability occurs for $V$ zero and small, but as $V$ is increased, both imaginary and real parts of the "unstable" eigenvalues decay, with the real parts decaying faster. Eventually the eigenvalues $\pm \lambda, \pm \lambda^{*}$ converge, pairwise, on the imaginary axis and the soliton stabilizes. Increasing $V$ still further, two of the resulting imaginary eigenvalues, $\lambda$ and $-\lambda$, start approaching each other. At $V=V_{c}$ where $\partial P / \partial V=0$, they collide and move onto the real axis. The soliton looses its stability once again - this time to a nonoscillatory mode. This scenario is exemplified by the curves $h=0.1$ and $h=0.15$ in Fig. 2 .

An interesting phenomenon occurs in the undriven case, $h=0$. As we mentioned, in this case the stationary solution of Eq.(2) is available in explicit form,
Eq.(12). We also noted that the associated momentum $P=V \sqrt{1-V^{2} / 4}$ has a maximum for finite $V=\sqrt{2}$. On the other hand, in any small- $h$ neighbourhood of this solution there are solitons $\psi_{+}$and $\psi_{-}$, of which $\psi_{+}$is stable only for $\partial P / \partial V>0$ (i.e. for $V<\sqrt{2}$ ) and $\psi_{-}$is stable only for $\partial P / \partial V<0$ (i.e. for $V>\sqrt{2}$ ). How can this be reconciled with the fact that the solution (12) is stable for all $V$ for which it exists $(V \leq 2)$ ?

The answer is related to the behaviour of the zero eigenvalue associated with the phase invariance of Eq.(2) with $h=0$. As $h$ increases from zero, this eigenvalue moves away from the origin. On the $\psi_{+}$branch, it passes onto the imaginary axis, while on the $\psi_{-}$branch, the eigenvalue moves onto the real axis instead.

\section{The travelling $\psi_{+}$soliton; $h>0.25$}

Let now $0.25<h<0.28$, and assume we are moving along the $\psi_{+}$branch in the direction of larger $V$. For small $V$ we have a quadruplet of complex eigenvalues $\pm \lambda, \pm \lambda^{*}$ implying the oscillatory instability. As $V$ is increased, both imaginary and real parts decay — as in the $h \leq 0.25$ case. However, this time imaginary parts decay faster than the real parts, and the two pairs of eigenvalues converge on the real axis. For velocities above this point the oscillatory instability is replaced by the translational instability. As $V$ is increased further, one pair of the newly born real eigenvalues grows in absolute value whereas the other pair decreases in magnitude. At the point $V=V_{c}$ where $P(V)$ reaches its maximum, the latter pair converges at the origin and moves onto the imaginary axis. (This does not render the soliton stable though, as the other pair remains on the real axis.) This scenario is exemplified by the curve $h=0.27$ in Fig. 2 .

Next, let $h$ be greater than 0.28 . For these $h$ the branch $P(V)$ emanating from the origin, turns back at some $V=V_{\max }$ (Fig.2), with the derivative $\partial P / \partial V$ remaining strictly positive for all $V \leq V_{\max }$. Below we will describe the transformation this solution undergoes when continued beyond the "turning point", while here we only wish to emphasize that no new zero eigenvalues can appear at this point. The reason is that $V=V_{\max }$ is a bifurcation point of solutions of the ordinary differential equation (3) but not of the partial differential equation (11)-(2). (In other words, $V$ is an "internal" parameter characterising the solution and not an "external" control parameter.) Indeed, the soliton is a member of a two-parameter $\left(x_{0}\right.$ and $V$ ) family of solutions of Eq.(11)-(2) and hence for any $V$ there are two zero eigenvalues in the spectrum of the linearised operator $\mathcal{H}$. Consequently, although being a turning point for the ODE (3), the value $V=V_{\max }$ is in no way special as far as the PDE (11)-(2) and its linearisation are concerned. No changes of the soliton's stability properties occur at this velocity.

How does one type of behaviour of the curve $P(V)$, occurring for $h>0.28$, replace the other one, arising 
for $h<0.28$ ? We scanned the interval $0.28000<h<$ 0.28020 and discovered a tiny region of transitional behavior, around $h=0.28005$. For this $h, P(V)$ grows until it reaches a maximum at $V_{c}=1.051$ and then starts decreasing, as in the case of $h<0.28$. However, the curve does not decay all way to $P=0$ as would be the case for $h<0.28$, but reaches a minimum at $V_{c c}=1.0563$. After that, the momentum starts growing, and, at $V_{\max }=1.0565$, the curve $P(V)$ turns back as for $h>0.28$ ! To give an idea of how small this window of transitional behaviour is, it suffices to say that for $h=0.28000$ the momentum $P(V)$ tends to 0 as $V \rightarrow c$, whereas for $h$ as close as 0.28010 , the curve $P(V)$ has a "turning point".

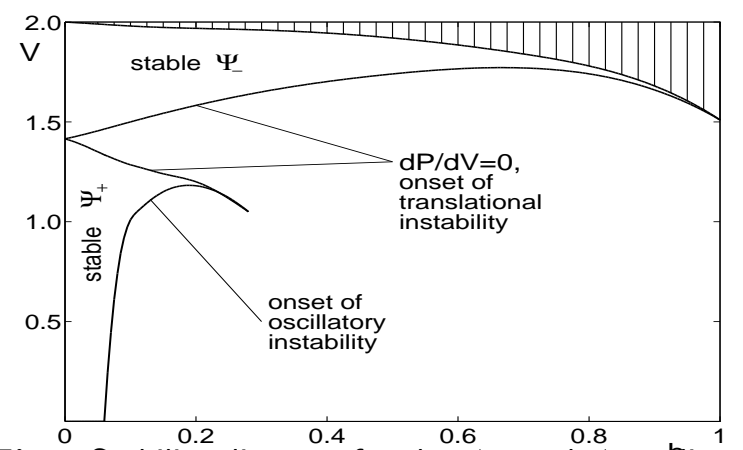

Fig.3 Stability diagram for the $\psi_{+}$and $\psi_{-}$solitons on the $(h, V)$-plane. In the region marked "stable" one of the two one-soliton solutions is stable whereas the other one is not. Across the solid line, the corresponding soliton looses its stability to an oscillatory or monotonically growing mode. No solitons exist in the dashed region.

What happens to the $\psi_{+}$soliton with $h>0.28$ (more precisely, with $h \geq 0.28010$ ) as we continue it beyond the turning point? Fig.4 (a) shows the momentum as a function of $V$. The point of intersection with the vertical axis $V=0$ corresponds to the twist solution (Eq. 22. with $z=\zeta$ given by Eq.(25).) On the diagram Fig.4, it is marked as $\psi_{T}$. As we mentioned before, the $(V=0)$ twist is a representative of a two-parameter family of stationary solutions of Eq.(2). Consequently, there should be four zero eigenvalues in the spectrum of the operator $\mathcal{H}$ in this case, with two linearly independent eigenfunctions given by $\left.\partial_{x} \Psi(z ; x)\right|_{z=\zeta}$ and $\left.\partial_{z} \Psi(z ; x)\right|_{z=\zeta}$. (Here $\Psi(z ; x)$ is a two-component vector formed by the real and imaginary parts of Eq.(22).) Numerically, we observed that as we approach the $(V=0)$-twist from the direction of positive $V$, a pair of opposite eigenvalues converges at the origin on the complex plane. The curve $P(V)$ does not have an extremum at this point and this may seem to be in contradiction with predictions of section II G. The paradox is resolved as soon as one recalls that the extremality condition $\partial P / \partial V=0$ was derived under the assumption that there is only one eigenvector associated with the zero eigenvalue whereas we have two linearly independent null eigenvectors in the case at hand.

As we continue further into the region $V<0$, the twist gives rise to a variety of multisoliton complexes; we shall describe them in the next subsection. Here we will restrict ourselves to the region $V>0$ where this branch can still be regarded as a branch of one-soliton solutions. Although these solutions undergo similar transformations for all $h$ in the interval $(0.28,1)$, there are a few differences with regard to the trajectories of eigenvalues on the complex plane. One difference worth to be mentioned is that for the driving strength $h=0.3$ and larger $h$, the quadruplet of complex $\lambda$ persists on the entire upper branch of $P(V)$ (i.e. for all $V>0$ ). This is in contrast to the case of $0.25<h<0.28$, where the complex quadruplet converges on the real axis. Near the left end of the interval $0.28<h<1$ (e.g. for $h=0.2802$ ), we have an intermediate pattern. Similarly to the case $h<0.28$, here the complex quadruplet converges on the real axis somewhere on the lower branch of $P(V)$ (i.e. before the turning point), but as we move onto the upper branch, the two emerging real pairs reunite quickly and the complex quadruplet reappears.

Next, as we know, there are only two points where a pair of eigenvalues can pass from the real onto the imaginary axis, or vice versa. One point is $V=V_{c}$ where $\partial P / \partial V=0$, and the other one is $V=0$. Therefore the dynamics of eigenvalues depends on which of the two points comes first, or, equivalently, whether the upper branch of $P(V)$ has the maximum for positive or negative $V$. For smaller values of $h$ in the interval $(0.28,1)$ (e.g. $h=0.3$ ), where $V_{c}>0$, two imaginary eigenvalues move to the real axis at $V=V_{c}$. These imaginary eigenvalues have detached from the continuous spectrum somewhere before the turning point (i.e. on the lower branch of $P(V)$.) The two newly born real eigenvalues first diverge from the origin but then reverse and, at $V=0$, move back onto the imaginary axis. For larger $h$ (e.g. $h=0.7$ ), where $V_{c}<0$, the pattern is different. Here, the two imaginary eigenvalues become real not at the point $V_{c}$ but at $V=0$. Subsequently, as we continue the branch to negative velocities, another pair of imaginary eigenvalues detaches from the continuum and at the point $V_{c}<0$ two (imaginary or real) eigenvalues pass through the origin.

Fig.3 shows the stability diagram of the $\psi_{+}$and $\psi_{-}$ solitons on the $(h, V)$-plane. For the $\psi_{+}$soliton, the range of stable velocities approaches $0 \leq V<\sqrt{2}$ as $h \rightarrow 0$, while the stability range of $\psi_{-}$tends to $\sqrt{2}<V \leq 2$. Finally, the domain of stability in the $h=0$ case is the union of the above two ranges: $0 \leq V \leq 2$.

\section{Other branches; $h>0.28$}

As we continue it to negative velocities, the twist (we are using this name here for $V \neq 0$-deformations of the quiescent twist solution) gradually transforms into a com- 
plex of two twists (plotted in Fig.5(a)). A further continuation of this branch takes us, via several "turning points", to a solution that can be interpreted as an association of the twist and two $\psi_{-}$solitons of opposite polarities (denoted $\psi_{(-T-)}$ ). This solution is depicted in Fig.5 (b).
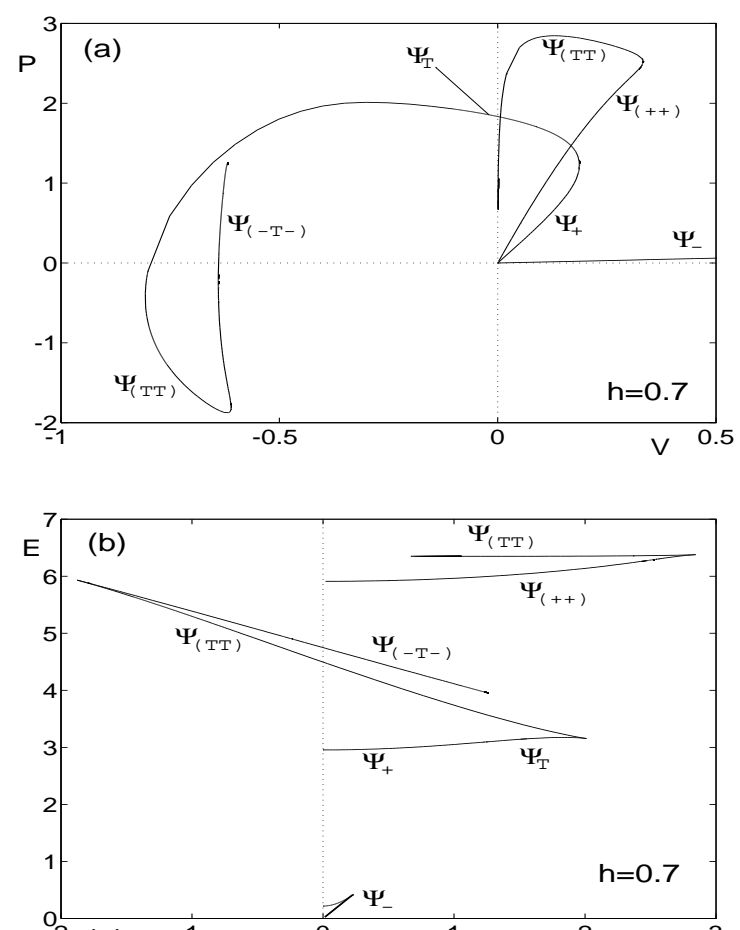

Fig.4 (a): The full bifurcation diagram for $h \mathrm{e}=0.7$. All branches shown in this figure are unstable. (For $h=0.7$ the soliton $\psi_{-}$is stable at large velocities but the stable region lies beyond the frame of this figure - see Fig.2.) More solution branches can be obtained by the reflection $V \rightarrow-V$, $P \rightarrow-P$. (b): The corresponding $E(P)$ dependence. The stable branch of $\psi_{-}$is depicted by a thick line at the bottom of the figure.

Fig.4 (b) shows the energy of different branches as calculated by Eq.(8). We have eliminated the dependence on the soliton's velocity between $P(V)$ and $E(V)$ to obtain $E$ as a function of $P$. The purpose of this "Legendre transformation" is the following. The stationary equation (3) can be regarded as a condition that the energy (7) be stationary under the fixed momentum (6): $(\delta E)_{P}=0$ or, equivalently, $\delta(E-V P)=0$, where $V$ is the Lagrange multiplier. Consequently, a steadily travelling soliton satisfies the relation of the Hamilton mechanics, $V=\partial E / \partial P$, and its velocity is given by the slope of the corresponding $E(P)$ branch in Fig.4 (b). The relation $\delta E=V \delta P$ implies that the curves $E(V)$ and $P(V)$ have extrema at the same set of points $V=V_{c}$ which will appear as cusps on the $E(P)$ plot. Since the function $P(V)$ has extrema precisely at points where linearised eigenvalues move from the imaginary to real axis, branches of the $E(P)$ curve separated by the cusps may have different stability properties. One such change of stabilities does indeed occur in Fig.4 (b) where the $\psi_{-}$-curve is seen to be partitioned into stable and unstable branch. Solitons on the stable branch have lower energies than unstable solitons with the same values of the momentum.
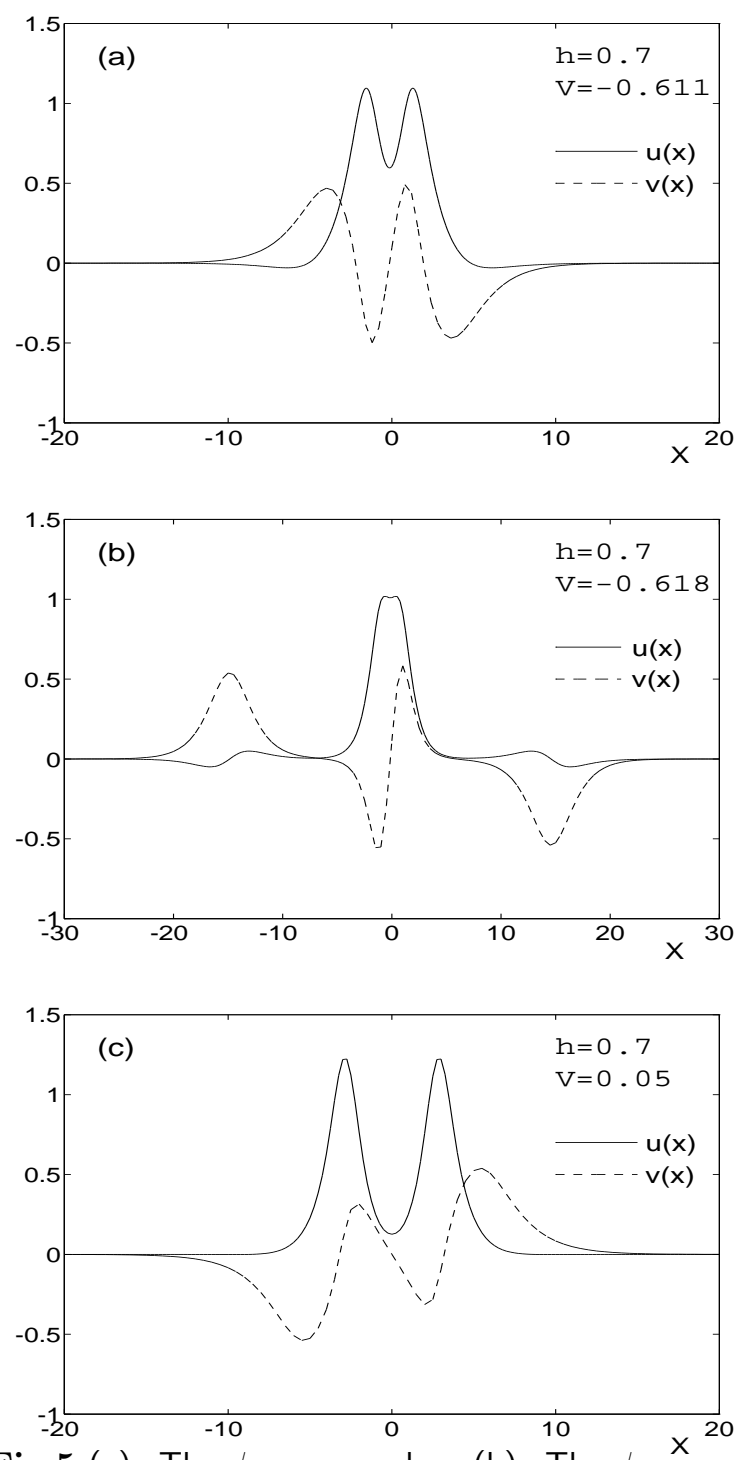

Fig.5 (a): The $\psi_{(T T)}$ complex. (b): The $\psi_{(-T-)}$ solution. (c): A complex of two twist solitons arising from the continuation of the $\psi_{(++)}$bound state. (Note the difference from the other two-twist complex shown in (a).) Solid line: real part; dashed line: imaginary part

Another branch emanating from the origin in Fig.4 (a), is a bound state of two solitons $\psi_{+}$. This solution was not obtained by the continuation from $V=0$ as Fig.4 may seem to be suggesting. Instead, we fixed a nonzero $V$ and continued in $h$ from the value $h=0.05$ where the complex $\psi_{(++)}$arises from the $V$-continuation of the twist soliton (see section IVE). Omitting details of this procedure, we start the description of the resulting branch at some point $(V, P)$ away from the origin. As we ap- 
proach the origin from this point, the separation between the solitons $\psi_{+}$in the complex $\psi_{(++)}$rapidly increases so that the field values between the two solitons become exponentially small. For example, for $h=0.7$, the (numerically calculated) separation at the point $V=0$ was equal to $z \approx 21$. The value of $|\psi|$ at the point on the $x$-axis, equally distanced from the left and right soliton, was of order $10^{-6}$. Consequently, the nonlinear term in the equation (3) becomes negligible away from the solitons' core and, in spite of an extremely small value of the residual that we used in our numerical algorithm $\left(10^{-10}\right)$, we were unable to distinguish between a genuine bound state and a linear superposition of two distant solitons. We conjecture that the complex $\psi_{(++)}$exists all way to $V=0$ but as $V \rightarrow 0$, the intersoliton separation $z \rightarrow \infty$. Another indication to this effect is that as $V \rightarrow 0$, the imaginary part of the solution tends to zero, rapidly and uniformly. For example, the imaginary part of the abovementioned numerical solution with the real part between the solitons $\geq 10^{-6}$, was smaller than $10^{-13}$ for all $x$. Since the only pure real solution that exists for $V=0$ is the (single) soliton $\psi_{+}$, the $V \rightarrow 0$ limit of the $\psi_{(++)}$complex should be an infinitely separated pair of the $\psi_{+}$'s.

If we, conversely, continue our solution away from the origin, the curve $P(V)$ turns left at some $V$ and the complex $\psi_{(++)}$transforms into what can be interpreted as a bound state of two twists (denoted $\psi_{(T T)}$ in Fig.4.) This solution is depicted in Fig.5(c). As $V \rightarrow 0$, the momentum of this bound state tends to zero (Fig.4 (a)). Unfortunately, we were only able to obtain this solution away from some small neighbourhood of $V=0$. (For $h=0.7$, the smallest value of the velocity for which we were still able to find the solution in question, was $V=0.000283$.) Whether this branch can be continued to $V=0$, remains an open question.

\section{E. Other branches; $h<0.28$}

As we have mentioned, for $h<0.28$ the branch $\psi_{+}$ extends all way to $V=c$ where it merges with the zero solution. No other solutions can be obtained from the $\psi_{+}$ soliton. However, in this case we can obtain new branches by continuing the (quiescent) twist soliton, Eq.(22) with $z=\zeta$.

It is convenient to start our description with the motionless twist solution with the negative momentum. As we move in the direction of positive $V$, the twist gradually transforms into a bound state of two $\psi_{+}$-solitons (see Fig.6 (a)). At some $V=V_{\max }$ the branch turns back, shortly after which, at the point $V=V_{c}$, the momentum reaches its maximum and starts decreasing. Adjacent to the turning point is a small range of velocities $V_{c} \leq V \leq V_{\max }$ where we have two stable solutions corresponding to each $V$. If we continue the branch with positive-momentum twist solution, also in the direction of positive $V$, the solution gradually transforms into a complex of two $\psi_{-}$solitons. The momentum reaches its maximum, starts decreasing, then the branch turns back in $V$ and we find ourselves approaching the origin on the $(V, P)$-plane (Fig.6 (a)). As we move towards the origin along the $\psi_{(++)}$or along the $\psi_{(--)}$branch, the separation between two solitons constituing the corresponding complex grows while the imaginary part of the solution tends to zero. Similarly to what we had for larger $h$ (section IVD), we conjecture that the separation becomes infinite at $V=0$ in both cases.
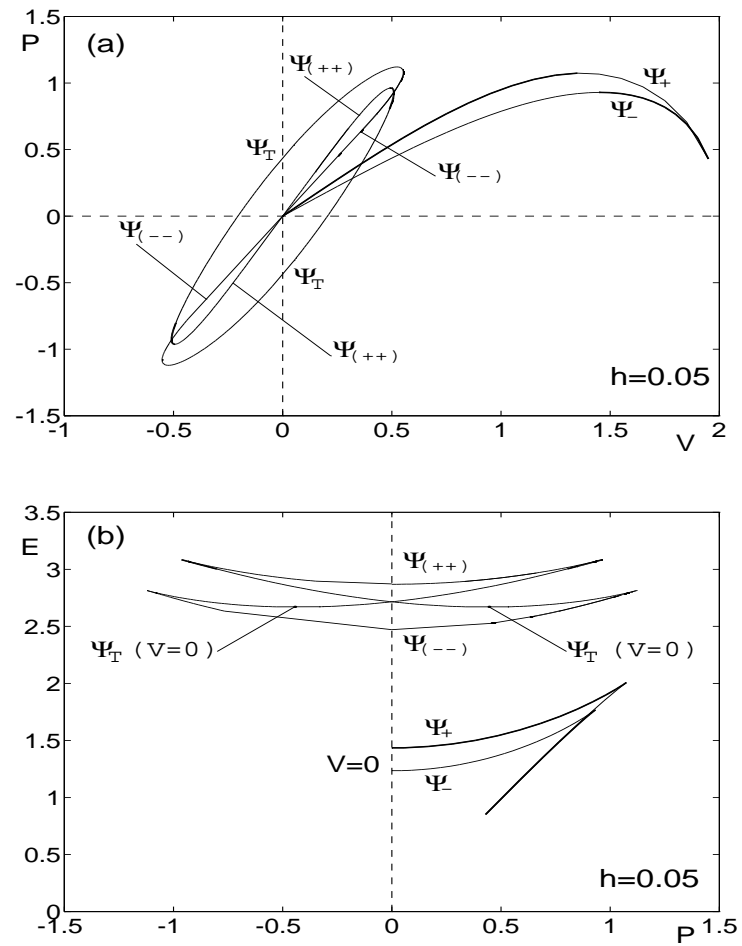

Fig.6 (a): The full bifurcation diagram for $h=0.05$. Thick respectively thin lines depict stable respectively unstable branches. Note a region of stability of the complex $\psi_{(++)}$. Additional branches can be generated by employing the reflection symmetry $V \rightarrow-V, P \rightarrow-P$. (b): The corresponding $E(P)$ diagram.

Fig.6 (b) shows the corresponding $E(P)$ dependence. As in Fig.4 (b), the cusps mark points of the zero crossing by the stability eigenvalues. Note that as in the case of large $h$ (Fig.4 (b)), there are solitons with the same value of the momentum but different energies. Similarly to Fig.4 (b), the stable branch of $\psi_{-}$has the lowest energy; bound states on the stable $\psi_{T} \rightarrow \psi_{(++)}$branch also have lower energies than their counterparts with the same $P$ and smaller $|V|$. However, in the case of the $\psi_{+}$ solitons we have an interesting reverse of fortunes: out of the two branches with the same $P$, the stable branch is the one with higher energy! 


\section{NONLINEAR STAGE OF INSTABILITY}

In this section we present results of our numerical simulations of the full time-dependent nonlinear Schrödinger equation (11) (with $\gamma=0$.) The objective was to study the nonlinear stage of the development of instabilities reported in the previous section and to identify the attractors emerging as $t \rightarrow \infty$. We utilised a split-step pseudospectral method, with $2^{11}=2048$ modes on the intervals $-40 \leq X \leq 40$ and $-80 \leq$ $X \leq 80$, and with $2^{12}=4096$ modes on the interval $(-60,60)$. The method imposes periodic boundary conditions $\psi(L / 2, t)=\psi(-L / 2, t), \psi_{X}(L / 2, t)=$ $\psi_{X}(-L / 2, t)$.

We have simulated the evolution of moving solitons unstable against an oscillatory mode and those with a positive, nonoscillatory, eigenvalue in their linearized spectrum. One of our conclusions here is that both types of instability give rise to the same asymptotic attractors. (This is in agreement with earlier simulations of motionless solitons 12.)

\section{A. The decaying breather}

Depending on the value of the driving strength, the initial conditions and the choice of the parameters of the numerical scheme, we observed one of the two scenarios. In the first scenario the soliton transforms into a bellshaped structure, with a small amplitude and large spatial width, oscillating approximately as $\psi \sim e^{i \omega t}$, with negative $\omega$. This localised solution was previously encountered in numerical simulations of Ref. 12 where it was termed breather. The amplitude of the breather slowly decays with time and the width slowly grows.

We have detected this scenario for the driving strength $h=0.1$, with the initial condition in the form of the $\psi_{+}$ soliton travelling with the velocity $V=0.05$ and with $V=0.8$. (For both values of the velocity the $\psi_{+}$soliton is unstable against an oscillatory mode.) Unlike earlier simulations [12] which started with the initial condition in the form of a quiescent unstable soliton and gave rise to a quiescent breather, the breather emerging from a travelling soliton has a nonzero speed.

One may naturally wonder whether the speed of the breather will decay to zero or approach a nonzero constant value as $t$ increases. Our simulations seem to support the latter hypothesis. In one run, the speed of the breather evolving out of the soliton travelling with the initial velocity of $V=0.05$, was seen to slowly grow and gradually approach the constant value of 0.1 . This simulation was repeated, with the same parameters of the numerical scheme and an initial condition which was only different from the previous one due to interpolation errors of order $10^{-6}$. In this run the breather was first seen to slow down, stop but then start moving in the opposite direction with the velocity close to -0.2 ; see Fig.7 (a).
(This remarkable sensitivity to the initial data deserves a separate comment; see below.) The velocity of the breather evolving out of the $V=0.8$ soliton, was tending to approximately 2.1. However, for large $t$ the unambigous interpretation of the numerical data is hindered by the growth of the amplitude of the radiation background. The radiation waves emitted by the oscillating breather re-enter the interval via the periodic boundary conditions and at a certain stage their amplitudes become comparable with the amplitude of the breather. Consequently, the constant-velocity motion of the breather may have been induced by the interaction with the backround radiations.
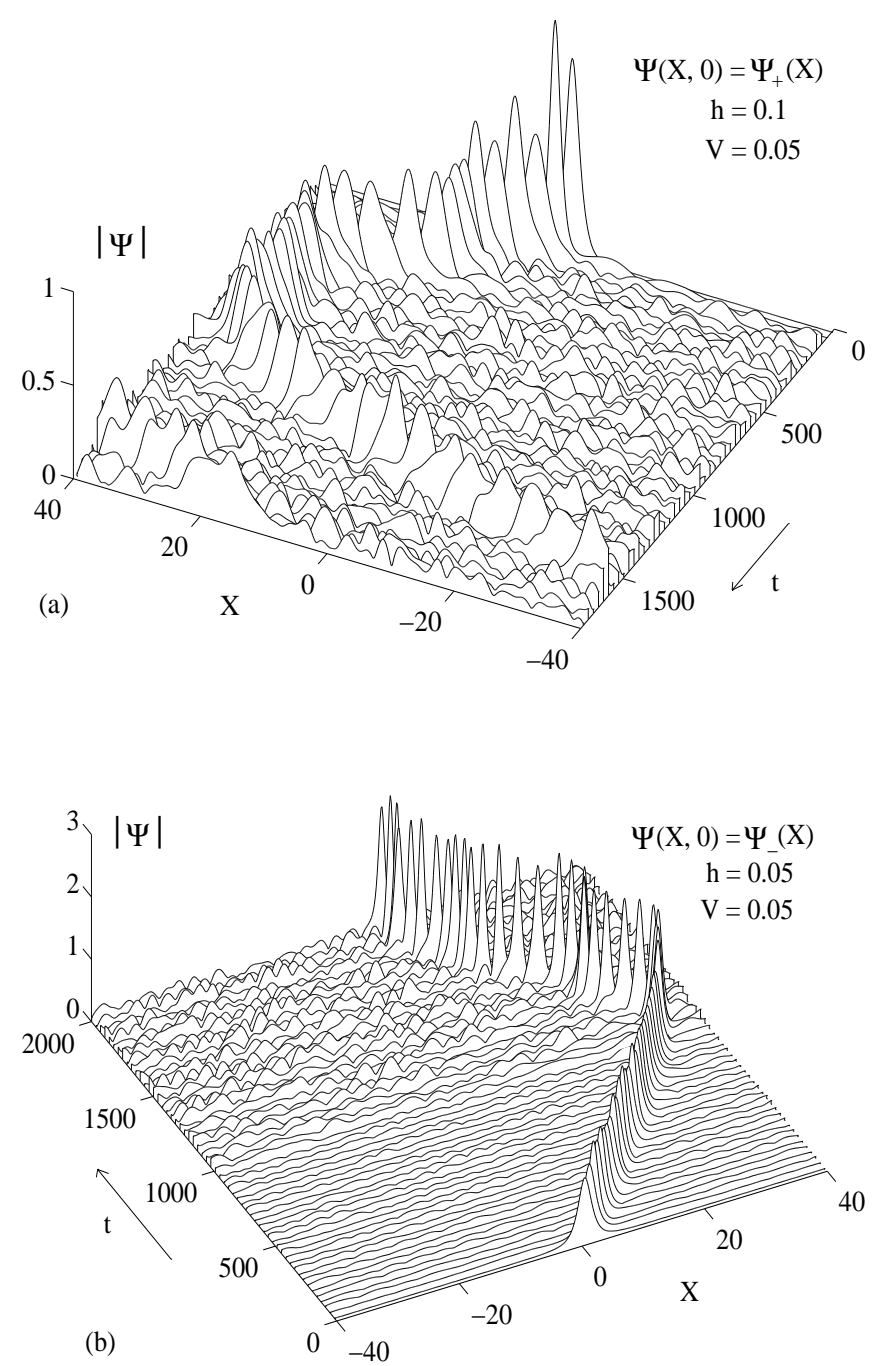

Fig.7 The two types of asymptotic attractors resulting from the decay of the unstable steadily travelling solitons: (a) the decaying and (b) the growing breather. (a) corresponds to $h=0.1$ and the initial condition in the form of the $\psi_{+}$soliton with $V=0.05$. In (b), $h=0.05$ and the ini-

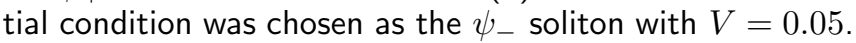
In both plots the emerging breather changes, spontaneously, its direction of motion. (Note that this happens not as a 
result of the reflection from the boundary, as the periodic boundary conditions are imposed.)

\section{B. The growing breather}

The decaying breather was detected in simulations on the interval $(-40,40)$ with $N=2^{11}$ modes. Changing the numerical parameters produced an entirely different scenario, however, - for the same value of the control parameter in Eq.(11) $(h=0.1)$, and for the same initial conditions $(V=0.05$ and $V=0.8)$.

Namely, we increased the number of the Fourier modes to $N=2^{12}$ and the length of the interval first to $L=120$ and then to 160. As in the case of $L=80$ and $N=2^{11}$, in simulations with the new values of $N$ and $L$ the unstable travelling soliton $\psi_{+}$was seen to transform into a bell-shaped structure, oscillating roughly as $\psi \sim e^{i \omega t}$. However, this time the emerging breather has a positive frequency $\omega$; its amplitude is large and continues to slowly grow, while the width is narrow and keeps on decreasing (Fig.7 (b)).

This attractor was also observed previously in 12. It was found there that the decaying and growing breather coexist. Whether the evolution of the same unstable soliton settles to one or the other asymptotic attractor, was found to depend on the choice of the phase of a small perturbation applied to the initial condition. In our present simulations, the perturbation is introduced simply by changing the parameters of the numerical scheme.

We also examined initial conditions in the form of translationally unstable solitons, including the $\psi_{+^{-}}$ soliton with $V=1.4$ for the driving strength $h=0.1$ and the $\psi_{-}$-soliton with initial velocities $V=0.05$ and $V=1.4$, for the driving strength $h=0.05$. For each of the above three situations the simulations were repeated with $2^{11}$ modes on the interval $-40 \leq X \leq 40$, and with $2^{12}$ modes on the intervals $(-60,60)$ and $(-80,80)$. In all nine runs the unstable soliton was seen to evolve into the growing breather. (It is quite likely that some other choices of the numerical parameters may give rise to the decaying breather instead.)

The velocity of the growing breather may vary during its evolution. It can even wander erratically, changing the direction of its motion several times, but eventually, for $t \sim 10^{4}$ or even earlier, the speed of the breather locks on to some constant value. Since the amplitudes of radiation waves are comparable with the amplitude of the breather at that stage, this effect can be induced by the breather-radiation interactions.

\section{CONCLUDING REMARKS AND OPEN PROBLEMS}

The main result of this paper is the demonstration of the existence of wide classes of travelling soliton solu- tions of the (undamped) parametrically driven nonlinear Schrödinger equation. We established the necessary conditions under which motionless solitons can be continued to nonzero velocities, and, in cases where these conditions were met, were indeed able to carry out the numerical continuation. The stability of all resulting branches of solutions was examined; oscillatory and translational instabilities identified, and the single-soliton stability chart compiled on the $(h, V)$-plane. The onset values of the translational instabilities, obtained numerically, were shown to verify the relation $\partial P / \partial V=0$ predicted by our theoretical analysis. As opposed to the case of the soliton $\psi_{-}$, which undergoes similar transformations for any $h$, the result of the continuation of the $\psi_{+}$has turned out to be sensitive to the value of the driving strength. We have identified two different transformation scenarios, one occurring for small and the other one for larger $h$, and described an interesting cross-over from one to the other.

In our analysis we paid a special attention to the trajectories of linearised eigenvalues on the complex plane. Apart from the information on the stability of different branches of solutions, the behaviour of the eigenvalues can give insight into the supercritical dynamics of solitons, i.e. dynamics beyond the instability threshold [12]. The motion of the eigenvalues in the undamped case that we are currently concerned with, allows one to even predict the asymptotic attractors arising when there is a small but nonzero damping in the system [12]. Relegating the corresponding bifurcation analysis to future publications, here we have restricted ourselves to a series of numerical simulations of the time-dependent NLS equation (11) (with $\gamma=0$.)

It is worth listing, separately, stable solutions obtained in this study. First, the quiescent soliton $\psi_{-}$, which is always unstable with respect to a nonoscillatory mode, stabilizes when travels faster than a certain critical velocity. The stability boundary satisfies $\partial P / \partial V=0$. Second, the quiscent soliton $\psi_{+}$is stable for $h<0.064$ and loses its stability to an oscillating soliton for $h>0.064$. For nonzero $V$ the stability region is shown in Fig.3. The lower boundary of this region corresponds to the onset of the oscillatory instability while along the upper boundary the soliton becomes unstable with respect to a nonoscillatory mode. The corresponding critical velocity satisfies $\partial P / \partial V=0$. Third, the bound state $\psi_{(++)}$also displays a region of stability for small $h$ - see Fig. 6 .

We conclude this section by pointing out to several open questions. In the first place, it would be interesting to continue the $\psi_{(-T-)^{-}}$-branch in Fig.4. Will this multisoliton complex keep on attaching more solitons on its flanks? Another "open-ended" branch $\left(\psi_{(T T)}\right)$ approaches the origin vertically down in Fig.4 (a); it would be interesting to continue this branch as well. The striking difference between the bifurcation diagrams for $h>0.28$ (Fig.4) and $h<0.28$ (Fig.6) is also worthy of a deeper analysis. Is the bifurcation diagram in Fig.6 
complete, or there are other multisoliton branches similar to those arising for large $h$ (Fig.4)? The next open question concerns the decaying and growing breather solutions arising as a result of the growth of the instability of steadily travelling solitons. If the radiations were prevented from re-entering the interval of simulation, would the velocity of the breather decay to zero or approach some nonzero value determined by the initial velocity of the unstable soliton? Finally, a challenging problem is to find out whether travelling solitons can exist in the presence of damping. The effect of dissipation will be, of course, to attenuate the soliton. It is not obvious whether the spatially uniform parametric pumping is capable of compensating this type of losses and sustaining the soliton's steady motion. It is fitting to note here that the parametrically driven, damped nonlinear Schrödinger equation has wide classes of stationary solitons [14], some of which may be continuable to nonzero $V$.

\section{ACKNOWLEDGMENTS}

We thank Dmitry Pelinovsky for drawing our attention to Ref. [13] and solution (22) presented therein. Useful conversations with Yuri Gaididei are also gratefully acknowledged. Special thanks go to Nora Alexeeva for providing us with a code for the time-dependent NLS equation and for her help with numerics. Finally, we are grateful to Professor Igor Puzynin for his continual encouragement and strong administrative support of this project. I.B. was supported by the NRF of South Africa and URC of the University of Cape Town. E.Z. was supported by an RFBR grant No.0001-00617.

$\dagger$ On leave from Department of Mathematics, University of Cape Town, Private Bag Rondebosch 7701, South Africa. Email: igor@maths.uct.ac.za

$\S$ On leave from the Laboratory for Computing Techniques, Joint Institute for Nuclear Research, Dubna, 141980, Russia. Email: elena@ultra.jinr.ru

¥ Email: baer@mpipks-dresden.mpg.de

[1] J.W. Miles, J. Fluid Mech. 148, 451 (1984); M. Umeki, J. Phys. Soc. Jpn. 60, 146 (1991); J. Fluid Mech. 227, 161 (1991)
[2] X.N. Chen and R.J. Wei, J. Fluid Mech. 259, 291 (1994); X. Wang, R. Wei, Phys. Lett. A 192, 1 (1994); W. Wang, X. Wang, J. Wang, R. Wei, Phys. Lett. A 219, 74 (1996); X. Wang, R. Wei, Phys. Lett. A 227, 55 (1997); X. Wang and R. Wei, Phys. Rev. Lett. 78, 2744 (1997); Phys. Rev. E 57, 2405 (1998); X. Wang, J. Acoust. Soc. Am. 104, 715 (1998)

[3] I.H. Deutsch and I. Abram, J. Opt. Soc. Am. B11, 2303 (1994); A. Mecozzi, L. Kath, P. Kumar, and C.G. Goedde, Opt.Lett. 19, 2050 (1994); S. Longhi, Opt.Lett. 20, 695 (1995); Phys. Rev. E 55, 1060 (1997)

[4] V.E. Zakharov, V.S. L'vov and S.S. Starobinets, Sov. Phys. Uspekhi 17, 896 (1975); M.M. Bogdan, A.M. Kosevich and I.V. Manzhos, Sov. J. Low Temp. Phys. 11, 547 (1985); H. Yamazaki and M. Mino, Prog. Theor. Phys. Suppl. 98, 400 (1989)

[5] I.V. Barashenkov, M.M. Bogdan, and V.I. Korobov, Europhys. Lett. 15, 113 (1991)

[6] L.N. Bulaevskii and V.L. Ginzburg, Sov. Phys. JETP 18, 530 (1964); J. Lajzerowicz and J.J. Niez, Journ. de Physique, 40, L165 (1979)

[7] A.V. Ustinov, B.A. Malomed, S. Sakai, Phys. Rev. B 57, 11691 (1998); H.S.J. van der Zant et al, Physica D 119, 219 (1998); O.M. Braun, Yu.S. Kivshar, Phys. Rep. 306, 1 (1998); A.C. Scott, Nonlinear Science. Emergence and Dynamics of Coherent Structures. Oxford Univ. Press (1999).

[8] P. Coullet, J. Lega, B. Houchmanzadeh, and J. Lajzerowicz, Phys. Rev. Lett. 63, 1352 (1990); C. Elphick, A. Hagberg, B.A. Malomed, and E. Meron; Phys. Lett. A 230, 33 (1997)

[9] I.V. Barashenkov and V.G. Makhankov, Phys. Lett. A 128, 52 (1988); I.V. Barashenkov, T.L. Boyadjiev, I.V. Puzynin, and T. Zhanlav, Phys. Lett. A 135, 125 (1989); M.M. Bogdan, A.S. Kovalev, and A.M. Kosevich, Sov. J. Low Temp. Phys., 15, 288 (1989); I.V. Barashenkov and E.Yu. Panova, Physica D 69, 114 (1993); Yu.S. Kivshar and X. Yang, Phys. Rev. E 49, 1657 (1994)

[10] I.V. Barashenkov, Phys. Rev. Lett. 77, 1193 (1996)

[11] D.E. Pelinovsky, Yu.S. Kivshar, and V.V. Afanasjev, Phys. Rev. E 54, 2015 (1996)

[12] N.V. Alexeeva, I.V. Barashenkov and D.E. Pelinovsky, Nonlinearity 12, 103 (1999).

[13] M.V. Tratnik and J.E. Sipe, Phys. Rev. A 38, 2011 (1988)

[14] I.V. Barashenkov and E.V. Zemlyanaya, Phys. Rev. Lett. 83, 2568 (1999); A. Il'ichev, Physica D 119, 327 (1998); I. Bakholdin, A. Il'ichev, Eur. J. Mech. B/Fluids, 18, 93 (1999) 